\title{
Short-Hairpin RNA-Mediated MTA2 Silencing Inhibits Human Breast Cancer Cell Line MDA-MB231 Proliferation and Metastasis
}

\author{
Jun Lu*, Mu-Lan Jin
}

\begin{abstract}
Objective: To observe the effects of metastasis-associated tumor gene family 2 (MTA2) depletion on human breast cancer cell proliferation and metastasis. Methods: A short-hairpin RNA targeting MTA2 was chemically synthesized and transfected into a lentivirus to construct $\mathrm{Lv}$-shMTA2 for infection into the MDA-MB231 human breast cancer cell line. At 48 hours after infection cells were harvested and mRNA and protein levels of MTA2 were determined by quantitative real-time polymerase chain reaction (qRT-PCR) and Western blotting, respectively. Cell viability and metastasis were assessed by CCK-8, wound-healing assay and Transwell assay, respectively. In addition, a xenograft model of human breast cancer was constructed to investigate cancerous cell growth and capacity for metastasis. Results: After infection with Lv-shMTA2, mRNA and protein levels of MTA2 was significantly reduced $(p<0.05)$ and MDA-MB231 cell proliferation and metastasis were inhibited $(p<0.05)$. In addition, mean tumor size was smaller than that in control group nude mice $(p<0.05)$ and numbers of metastatic deposits in lung were lower than in control group mice $(p<0.05)$. Depletion of MTA2 affected MMP-2 and apoptosis-related protein expression. Conclusions: For the first time to our knowledge we showed that MTA2 depletion could significantly inhibit human breast cancer cell growth and metastasis, implying that MTA2 might be involved in the progression of breast cancer. The role of MTA2 in breast cancer growth and metastasis might be linked with regulation of matrix metalloproteinase and apoptosis.
\end{abstract}

Keywords: shRNA - MTA2 - breast cancer - growth - metastasis

Asian Pac J Cancer Prev, 15 (14), 5577-5582

\section{Introduction}

Breast cancer is a worldwide threat for female health. Its incidence and mortality rates are increasing rapidly. According to statistics, breast cancer is the fifth most common cause of cancer death worldwide, as it comprises of $10.4 \%$ of all cancer incidence thus making it the most common type of non-skin cancer in women. Worldwide 519,000 deaths were caused by breast cancer (7\% of cancer deaths) in 2004 (Shaukat et al., 2013). Metastasis is a major concern for breast cancer prognosis (Shaukat et al., 2013), especially in developing countries where cancers are always diagnosed in the late stages (Wu et al., 2014). Hence, better understanding of molecular mechanisms underlying breast cancer metastasis is still required.

Breast cancer is a complex and multistep disease involving the co-ordinal interaction of multiple genes and the accumulation of multiple molecular and morphologic changes within a cell (Zhang et al., 2006). To date, one breakthrough in transcription regulation is the discovery of nucleosome remodeling and histone deacetylation (NuRD) complex which is critical for transcriptional regulation of cancer-related genes (Roberts and Orkin, 2004; Cho et al., 2004). Notably, metastasis-associated protein (MTA) family is the functional components of NuRD complex. In vertebrates, three members are identified in MTA family: MTA1, MTA2 and MTA3 among which MTA1 and MTA3 were mostly reported.

MTA1 was the first found member and later reported to be widely expressed in various cancers, including breast cancer (Toh et al., 1994), esophageal carcinomas (Toh et al., 1999), and gastrointestinal carcinomas (Toh et al., 1997). In mammary adenocarcinoma, MTA1 was detected to be differentially expressed in highly metastatic cell lines (Toh et al., 1994). Similarly, MTA1 confers metastasis-promotion effect on esophagus carcinoma as well as gastrointestinal carcinomas (Toh et al., 1997; Toh et al., 1999). However, several normal mouse tissues and organs also express substantial levels of MTA1. Thus, MTA1 may play a role in both the physiologic and the pathologic states of cells (Kumar et al., 2003). Furthermore, regulatory mechanism underlying MTA1promoted invasion and migration was also examined. In breast cancer, MTA1 could structurally interact with ER 
and directly repress ER expression, leading to inhibition of its transactivation function (Kumar, 2003). Conversely, MTA3 exhibits a unique regulation function to ER by indirectly regulating ER target genes (Fearon, 2003; Kumar, 2003). In addition, MTA3 is also a repressor of snail (Fujita et al., 2003) and is lost in the polyoma virus middle-T mammary oncogene model of mammary cancer, while MTA1 and MTA2 expression is retained (Zhang et al., 2006), indicating functionally specialized activity for MTA1 and MTA3.

MTA2 is structurally homologous to MTA1. MTA2 was reported to be overexpressed in cervical cancer and correlated well with rapid cell division (Matsusue et al., 2001). Recently, it was also evidenced that MTA2 promoted gastric cancer cell invasion (Zhou et al., 2013). However, the role of MTA2 in breast cancer proliferation and metastasis remains unclear. To this end, we firstly stably silenced MTA2 in breast cancer cell line MDAMB231 cells by infection with lentivirus expressing green fluorescent protein (GFP)-shMTA2 (Lv-shMTA2) or GFP (for control, Lv-shNC). We then examined effects of MTA2 ablation on MDA-MB231 cell proliferation and metastasis.

\section{Materials and Methods}

\section{Cells and cell culture}

Human breast cancer cell line MDA-MB231 cells were purchased from American Type Culture Collection (ATCC, Manassas, VA, USA). Cells were maintained in RPMI 1640 (Gibco, MD, USA) containing 10\% fetal bovine serum (FBS) (Gibco) at $37^{\circ} \mathrm{C}$ in humidified atmosphere of $5 \% \mathrm{CO} 2$.

\section{Reagents}

Mouse anti-human MTA2 antibody, mouse antihuman Actin antibody and secondary antibody were all purchased from Santa Cruz (CA, USA). Specific shRNA targeting MTA2 (shMTA2) was commercially from Santa Cruz (CA, USA). For control, a negative control shRNA (shNC) was also used. Matrigel was purchased from BD Biosciences (San Jose, CA, USA). Cell counting kit-8 (cck-8) was purchased from Dojindo (Japan).

\section{Quantitative real-time PCR ( $q R T$-PCR)}

Total RNA of cell lysates were extracted with Trizol solution (Invitrogen, Carlsbad, CA, USA) at $24 \mathrm{~h}$ postinfection and reverse transcribed to cDNA using a PrimerScript RT reagent Kit (TAKARA, Shiga, Japan) in accordance with the manufacturer's instructions. $\beta$-actin was used as a normalization control. Primers were chemically synthesized by Sangon Biotechnology (Shanghai, China). Real-time RT-PCR primers were as follows:

MTA2, forward 5'-TGTACCGGGTGGGAGATTAC-3', reverse 5'-TGAGGCTACTAGAAATGTCCCTG-3'; $\beta$-actin, forward 5'-TGGCACCCAGCACAATGA A-3', reverse 5'-CTA AGT CAT AGT CCG CCT AGAAGC A-3' Real-time PCR was performed using SYBR Premix EX Tag (TAKARA, Shiga, Japan) on an ABI 7500 thermal cycler (Applied Biosystems). The PCR conditions were as follows: PCR cycle conditions were $95^{\circ} \mathrm{C}$ for $30 \mathrm{~s}$, and 40 cycles of $95^{\circ} \mathrm{C}$ for $5 \mathrm{~s}$ and $60^{\circ} \mathrm{C}$ for $34 \mathrm{~s}$. All experiments were performed in triplicate and Relative mRNA was determined by using the formula $2-\Delta \mathrm{CT}$ (CT; cycle threshold $)$ where $\Delta \mathrm{CT}=\mathrm{CT}($ target gene $)-\mathrm{CT}(\beta$-actin $)$.

\section{Western blot}

Cells were harvested $48 \mathrm{~h}$ after infection in each group and protein concentration was determined using the Bio-Rad DC protein Assay (Bio-Rad, CA, USA). Subsequently, 30 micrograms protein were fractionated by $10 \%$ SDS-PAGE gel and transferred to PVDF membranes. PVDF membranes were blocked by $5 \%$ skim milk for 1 $\mathrm{h}$, then were incubated overnight at $4^{\circ} \mathrm{C}$ with primary antibodies, including MTA2 (1:1000) and $\beta$-Actin (1:500). Goat anti-mouse secondary antibodies (1:3000) and enhanced chemoluminescent autoradiography (ECL kit, Amersham) was used to visualize the protein bands.

\section{Cell Counting Kit (CCK-8) proliferation assay}

To detect cell viability, 3-(4, 5-dimethylthiazol-2-yl)-2, 5-diphenyltetrazolium bromide (MTT) colorimetric assay was performed using CCK-8 kit. Briefly, MDA-MB231 cells were washed with PBS and suspended at a final concentration of $2 \times 104$ per $\mathrm{ml}$ in an assay medium and dispensed into 96-well plates. The plates were incubated at $37^{\circ} \mathrm{C}$ for 5 days in a humidified $\mathrm{CO} 2$ incubator. At each monitored time point (1, 2, 3, 4 and 5 day), 10 $\mu$ l CCK-8 was added to each well. Then plates were incubated at $37^{\circ} \mathrm{C}$ for $2 \mathrm{~h}$. The absorbance at $595 \mathrm{~nm}$ was measured using a synergy 2 multi-mode microplate reader (Bio Tek Instruments, Winooski, VT, USA). Absorbance in Lv-shNC group was set as 1 on day 1 . For the graph in Figure 3, data were expressed as relative value normalized to that in Lv-shNC group on day 1. Experiments were performed in triplicate.

\section{Wound-healing assay and Transwell assay}

For wound-healing assay, MDA-MB-231 cells plated on 6-well plates were grown to form a confluent monolayer, and wounds were made with sterile pipette tips. Photographs were taken at indicated time points after wounds were made. For invasion assay, $100 \mu \mathrm{l}$ of diluted Matrigel (BD Biosciences) was used to coat the chambers' membrane ( $8 \mu \mathrm{m}$ for 24 -well plate, Millipore). Fifty thousand cells in $100 \mu$ l serum-free medium were added into the upper chambers, with full medium in lower chambers, then, cultured for $48 \mathrm{~h}$. For migration assay, cells were cultured for $24 \mathrm{~h}$ under the same conditions without Matrigel. Cells were fixed by $10 \%$ formalin, and stained by Giemsa, and were photographed and counted by inverted microscope.

\section{Mouse xenograft breast cancer models}

MDA-MB231 cells pre-treated with either LvshMTA2 or Lv-shNC were harvested and $5 \times 106$ cells in $200 \mu \mathrm{l}$ of phosphate-buffered saline were subcutaneously injected into the mammary fat pad of 6-week-old female BALB/c mice ( $\mathrm{n}=$ for each group). Tumor size was measured in two dimensions with calipers twice a week, 
A: Lv-shNC
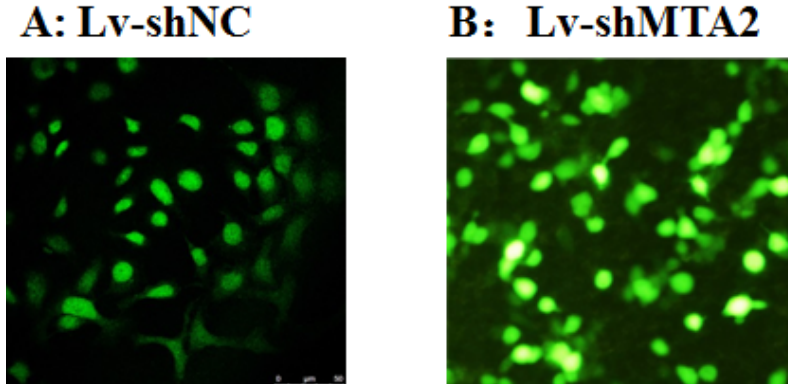

Figure 1. Infection was Efficient in both $\mathrm{Lv}$-shNC and Lv-shMTA2 Groups. (A) MDA-MB231 cells were mostly (approximately 90\%) infected with lentivirus expressing only GFP. (B) MDA-MB231 cells were mostly (approximately 90\%) infected with lentivirus expressing GFP and shRNA against MTA2.

up to 30 days after injection. Tumor volume (v) was calculated according to the formula $\mathrm{V}=\mathrm{L} \times \mathrm{W} 2 / 2(\mathrm{~L}$ represents length while $\mathrm{W}$ represents width). All efforts were made to minimize suffering.

\section{Statistical analysis}

Data were expressed as mean \pm SD of three independent experiments in which each assay was performed in triplicate. Independent samples t test was used in quantitive data analysis. P-value $<0.05$ was considered as statistical significant.

\section{Results}

Specific depletion of MTA2 in breast cancer MDAMB231 cells.

To address the question whether MTA2 was critically involved in breast cancer cell proliferation and metastasis, we employed the RNA interference technique to knockout the expression of MTA2 in breast cancer cell line MDAMB231 cells. We infected MDA-MB231 cells with lentivirus stably silencing MTA2 (Lv-shMTA2). As a control, we also construct a negative control lentivirus (Lv-shNC). Meanwhile, lentivirus used here could express green fluorescence (GFP), making it easy for us to evaluate the infection efficiency of each group. As shown in Figure 1, most cells were infected with corresponding lentivirus in both Lv-shNC and Lv-shMTA2 groups. Infection efficiency in both groups was nearly $90 \%$ as evidenced by the GFP signals. We then detected mRNA level and protein level of MTA2 in both groups. As expected, we found that mRNA level of MTA2 were significantly reduced when cells were infected with Lv-shMTA2 ( $p<0.05$, Figure 2A). Concomitantly, band density in western blot analysis was also greatly lowered in Lv-shMTA2-infected MDA-MB231 cells as compared with that in Lv-shNC-infected cells (Figure 2B). These results suggested that the lentivirus stably silencing MTA2 was successfully constructed in MDA-MB231 cells.

Depletion of MTA2 inhibits cell growth in MDA-MB231 cells

To examine the effects of MTA2 ablation on cell proliferation, we performed CCK-8 assay to continuously

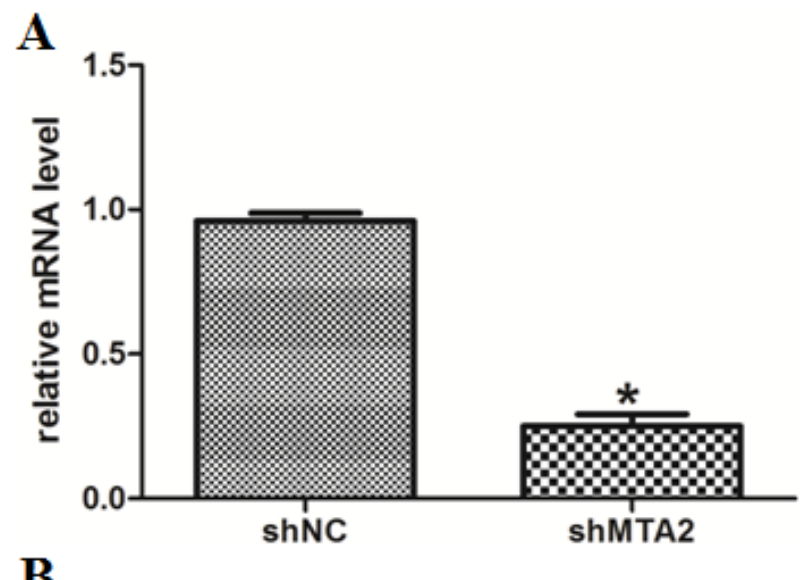

\section{Lv-shNC Lv-shMTA2}

MTA2

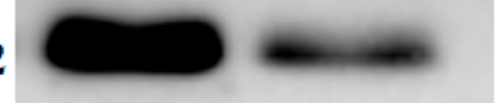

$\beta$-actin

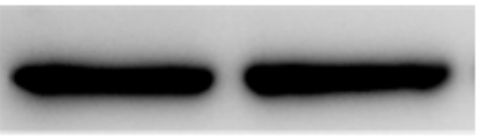

Figure 2. Expression Levels of MTA2 were Efficiently Depleted after Infection with Lv-shMTA2. (A) mRNA level of MTA2 was significantly reduced after infection with Lv-shMTA2; $* p<0.05$. (B) Protein level of MTA2 was significantly reduced after infection with Lv-shMTA2.

monitor cell numbers in each group. Absorbance which is an indicator of cell viability was measured at each monitored time point. We found that cells infected with non-specific shRNA could retain a high proliferative rate in all the five time points (Figure 3A, Lv-shNC group). However, cells infected with specific shRNA targeting MTA2 were significantly impeded from duplication (Figure 3A, Lv-shMTA2 group). Actually, mean absorbance in Lv-shMTA2 group was only $1.45 \pm 0.10$, compared with $2.92 \pm 0.16$ in $\mathrm{Lv}$-shNC group on day 5 $(p<0.05)$. Cell proliferative rate was nearly half slowed down. In parallel, we also performed colony formation assay and found that cells infected with Lv-shMTA2 exhibited lower colony formation ability (Figure 3B, left panel). Quantification of the colonies showed that shMTA2 could inhibit MDA-MB231 cells colony formation by up to $66 \%$ (Figure $3 \mathrm{~B}$, right panel). Our results indicated that knockdown of MTA2 could inhibit cell growth in MDA-MB231 cells.

Depletion of MTA2 inhibits cell migration and invasion in MDA-MB231 cells

Furthermore, as an approach to study migration, wound-healing assay was then conducted. As shown in Figure 3C, apparent wound could be seen $12 \mathrm{~h}$ post infection in both Lv-shNC and Lv-shMTA2 groups. However, 24h post infection, MDA-MB231 cells depleted of MTA2 were significantly impaired in wound recovery, whereas the control cells had almost closed the wound. To further assess the effects of MTA2 knockout 

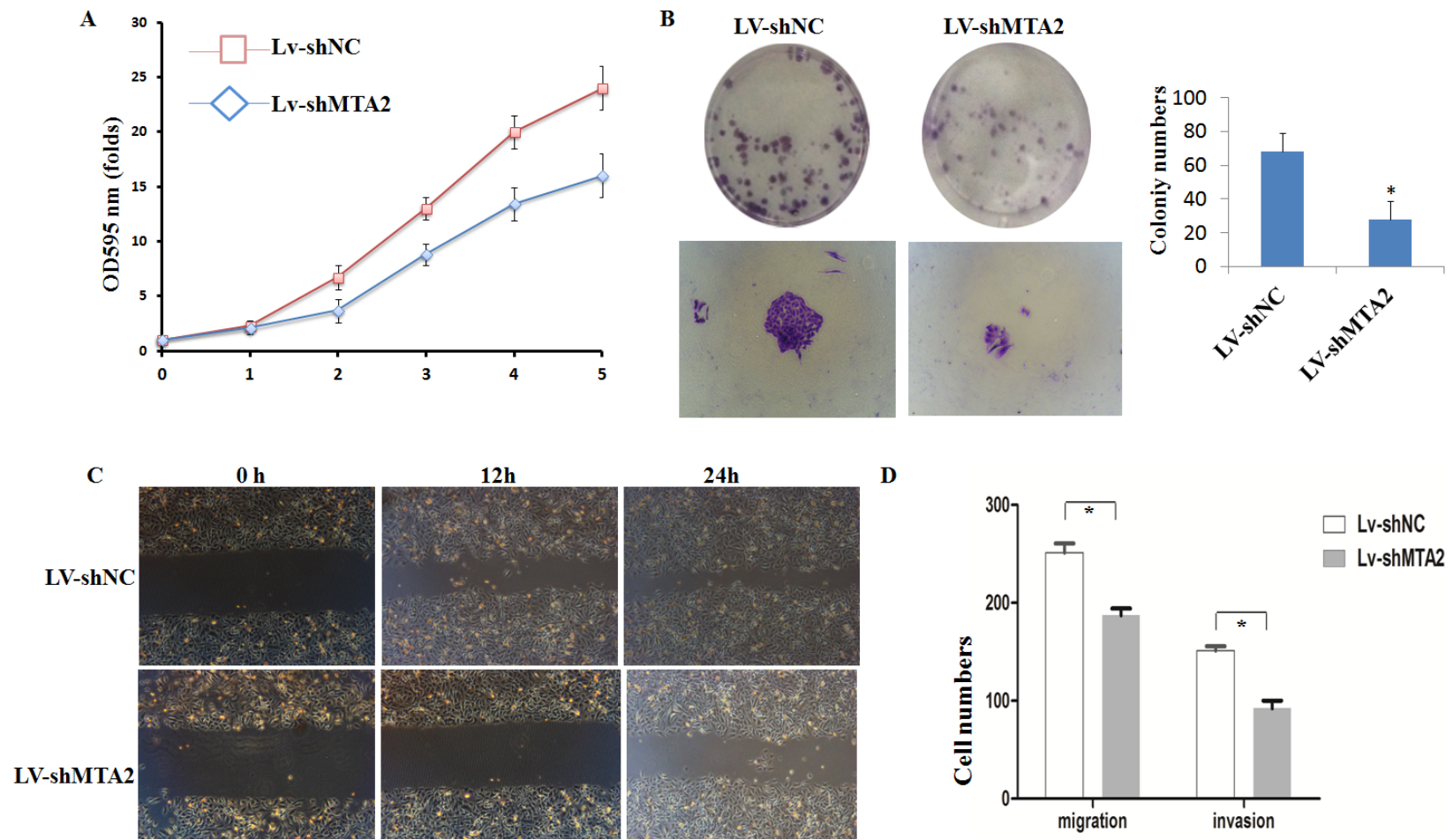

$\square$ Lv-shNC

LV-shMTA2

Figure 3. Cell Proliferation, Migration and Invasion were Inhibited after MTA2 Depletion in MDA-MB231

Cells. (A) Cell numbers were continuously monitored on day 1,2,3,4 and 5, respectively with CCK-8 kit. (B) Colony formation assay was conducted to assess cell colony-formation ability after MTA2 depletion. (C) A wound-healing assay was performed to study cell migratory ability in response to MTA2 depletion. Wound recovery was photographed every $12 \mathrm{~h}$ post infection. (D) Migrated cells in migration assay and invasion assay were stained and counted in LvshNC and Lv-shMTA2 groups; ${ }^{*} p<0.05$

Table 1. Depletion of MTA2 Inhibits Migration and Invasion in MDA-MB231Cells

\begin{tabular}{lcc}
\hline group & $\mathrm{CNs}^{\#}$ (migration assay) & $\mathrm{CNs}^{\#}$ (invasion assay) \\
\hline Lv-shNC & $251.0 \pm 9.6$ & $187.0 \pm 7.2$ \\
Lv-shMTA2 & $151.0 \pm 4.5^{*}$ & $92 \pm 8.2^{*}$ \\
\hline
\end{tabular}

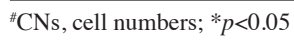

on cell migration and invasion, we performed transwell assay. Cells were pretreated with either Lv-shNC or LvshMTA2 and then migrated cells were photographed in five fields and counted. In this way, average migrated cells in each group were calculated. In migration assay, average 151 cells in Lv-shMTA2 group were observed to transmigrate to lower chamber while average 251 cells did that in Lv-shNC group (Figure 3D, Table $1,{ }^{*} p<0.05$ ). Similarly, in invasion assay, cells transmigrated to lower chamber were significantly decreased after infection with Lv-shMTA2. Actually, migrated cells infected with LvshMTA2 (92 \pm 8.2$)$ counts for only half of those infected with non-specific shRNA (187.0 \pm 7.2) (Figure 3D, Table 1, $\left.*_{p}<0.05\right)$. Altogether, knockout of MTA2 in MDA-MB231 cells caused a notable decrease in migrated cells.

Depletion of MTA2 inhibits tumor growth and metastasis in vivo

Next, we construct a mouse model bearing breast cancer with each group of mice injected with equal amounts of MDA-MB231 cell suspension. Eight mice were divided into two groups: Lv-shNC-infected group and Lv-shMTA2-infected group ( $\mathrm{n}=4$ for each group). Tumor volume was observed twice a week and after 30 days, all the mice were sacrificed. Tumors were dissected and photographed. As expected, we observed a smaller tumor size in the Lv-shMTA2 group as compared with that in $\mathrm{Lv}$-shNC group (Figure 4A). When dissecting, we also observed metastatic nodules in lung. We found that 3 out of 4 mice in $\mathrm{Lv}$-shNC group exhibited metastatic nodules in lung while only 2 out of 4 mice in Lv-shMTA2 group exhibited metastatic nodules in lung (Figure 4B). Nodule numbers in lung were also counted. Average 31.5 nodules were presented in Lv-shNC group while only average 3 nodules in lung were presented in Lv-shMTA2 group (Figure 4B). In accordance with these data, histology analysis of lung tissues from the two groups also showed that control cells exhibited metastatic nodule (arrow indicated), whereas MTA2-depleted MDA-MB231 cells failed to exhibit metastatic nodule in lung (Figure 4C). Our observations indicated that knockout of MTA2 could slow down tumor growth and impede tumor metastasis.

MTA2 knockout affected MMP-2 and apoptosis-related protein expressions

To uncover the mechanisms underlying MTA2mediated breast cancer growth and metastasis, we performed western blot analysis. Matrix metalloproteinase (MMPs) were critical factors involved in tumor metastasis (Fagan-Solis et al., 2013; Puzovic et al., 2014; Radenkovic et al., 2014). Apoptosis was also an important process in tumor cell proliferation (Armania et al., 2013; Choi and Kim, 2013). We randomly selected several proteins, including MMP-2, MMP-9, anti-apoptotic protein BCL$\mathrm{XL}$ and the pro-apoptotic protein BAS. As revealed 

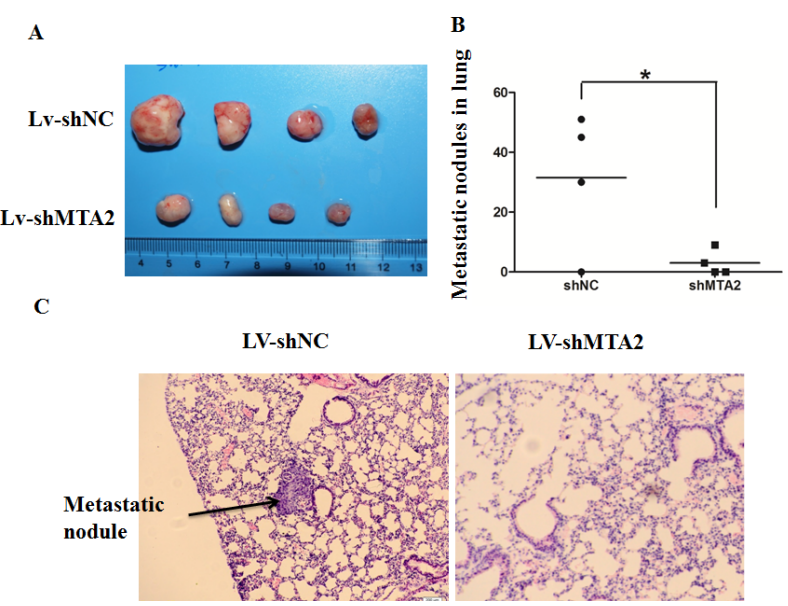

Figure 4. Tumor Growth and Metastasis were Significantly Impeded after MTA2 Depletion in Vivo. We Constructed a Mouse Model of Breast Cancer. (A) On day 30 when mice were sacrificed, average tumor volume in MTA2 depletion group was significantly smaller than that in control group. (B) Metastatic nodules in lung in MTA2 depletion group were significantly reduced compared with those in control group. ${ }^{*} p<0.05$. (C) Histology analysis of lung tissues from control and LvshMTA2-infected mice. Arrow indicated one metastatic nodule in the lung.

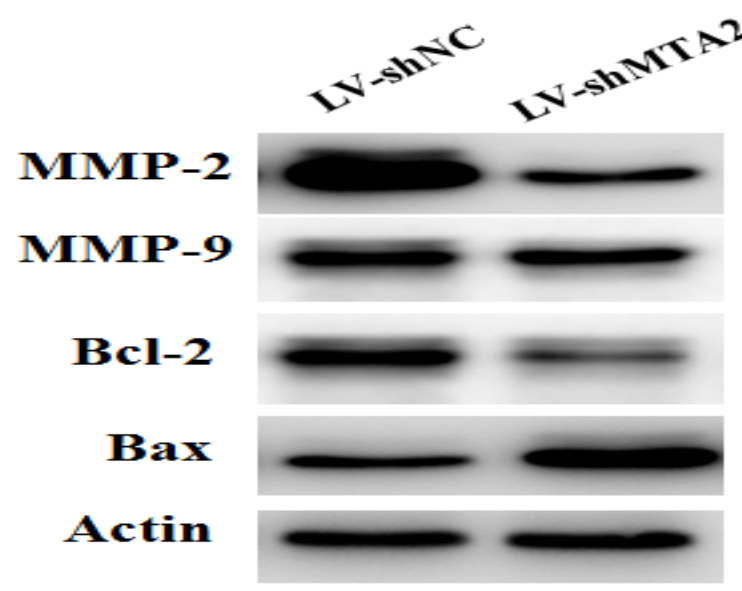

Figure 5. Depletion of MTA2 Affected MMP-2 and Apoptosis-Related Protein Expressions. MDA-MB231 cells were infected with Lv-shNC or Lv-shMTA2, respectively. MMP-2 and anti-apoptotic protein BCLXL were decreased in Lv-shMTA2 group, whereas pro-apoptotic protein BAS was inversely increased in Lv-shMTA2 group.

in Figure 5, we found that knockout of MTA2 caused apparent decrease in MMP-2 and anti-apoptotic protein BCL-XL expressions, whereas the pro-apoptotic protein BAS was increased in response to MTA2 depletion. In addition, MMP-9 was not affected at all by MTA2 expression manipulation. Hence, MTA2-mediated breast cancer cell growth and metastasis might be linked with matrix metalloproteinase and apoptosis process.

\section{Discussion}

The majority of breast cancer patients succumb to complications from distant metastases rather than the primary tumor (Fidler, 1990; Erdogan et al., 2014). Thus understanding the molecular processes that control breast cancer progression provides the foundation for early therapeutic intervention. In this study, we demonstrated that MTA2 silencing mediated by short-hairpin RNA (shRNA) could inhibit breast cancer cell line MDAMB231 cell proliferation. Compared with cells infected with Lv-shNC, cells infected with specific shRNA targeting MTA2 in MDA-MB231 cells exhibited a slower proliferative rate. We then examined the effects of MTA2 depletion on MDA-MB231 cell migration and invasion in transwell assay. We found that cells infected with the negative control lentivirus retained a high transmigration to the lower chamber. However, cells infected with lentivirus stably silencing MTA2 by specific shRNA were significantly hindered from migration to lower chamber. In addition, we construct a mouse model to assess the effects of MTA2 depletion on breast tumor growth and metastasis in vivo. Concomitantly, we observed a smaller average tumor size and decreased metastatic nodules in lung in the Lv-shMTA2 group mice. Taken together, it could be concluded that knockdown of MTA2 could significantly inhibit breast cancer cell growth and metastasis.

Herein we used MDA-MB231 cells to investigate the role of MTA2 in breast cancer proliferation and metastasis. MDA-MB231 is an invasive cell line in breast cancer. So we employed RNAi technique to deplete MTA2 expression to assess the changes of MDA-MB231 cell phenotypes, including proliferation, migration and invasion abilities. Furthermore, in our attempt to uncover the mechanisms underlying MTA2-mediated breast cancer progression, we observed that MMP-2 and anti-apoptotic protein BCL$\mathrm{XL}$ were decreased, whereas pro-apoptotic protein BAS was increased in response to MTA2 depletion (Figure 5). Our data indicated that the role of MTA2 in breast cancer might be related to matrix metalloproteinase and apoptosis process. In other words, MTA2 might promote breast cancer cell proliferation through regulating apoptosis process. And MTA2-promoted breast cancer metastasis might be attributed to elevated MMP-2 expression. Though the detailed mechanisms how MTA2 affected these protein expressions remain elusive, one clue is that MDA-MB231 is an ER negative cell line in breast cancer. Hence, we could at least speculate that the regulatory mechanism contributing to MTA2-induced breast cancer cell growth and metastasis is possibly detoured from ER pathway due to the ER negative characteristic of MDA-MB231 cells. In fact, MTA2 was reported to be a repressor of ER whose overexpression leads to estrogenindependent growth of human breast cancer cells (Cui et al., 2006). This report was, at least partially, in support of our hypothesis.

Instead, we speculate that the regulatory mechanism underlying MTA2-induced cell phenotype changes may be linked with transcription regulation of downstream genes other than ER. As a fundamental component of NuDR complex which is a major macromolecular form of histone deacetylases (Tong et al., 1998), MTA2 probably confers transcription regulation to target genes by functional protein-protein interaction and/or indirectly deacetylate histone in which way gene transcription was 
strictly controlled. One example is that MTA2 has been proposed to interact with p53 and such interaction plays an important role in tumor progression (Luo et al., 2000). Interactions with other transcription regulators might be conceivable that contribute to the MTA2-mediated matrix metalloproteinase and apoptosis-related protein expressions. Additionally, the role of MTA2 in breast cancer metastasis might also be linked with microRNAs, because six microRNAs were reported to regulate multiple and various steps of the metastasis cascade by targeting metastasis-associated genes (Ding et al., 2014). In all, more work needs to be done as to the network between MTA2 and breast cancer metastasis.

In this study, we presented that knockdown of MTA2 by specific shRNA delivered by lentivirus caused significant inhibition of cell proliferation, migration, invasion in breast cancer MDA-MB231 cells. And tumor size as well as metastatic nodules in lung was also notably limited in MTA2 depletion cells in a mouse model of xenograft. In all, we propose that MTA2 might be a critical factor involved in breast cancer progression. Specific therapies targeting MTA2 might provide novel clues for development of clinical interventions for breast cancer treatments and prevention.

\section{Acknowledgements}

This study was supported by Shengyang Science and Technology Project Plan.

\section{References}

Armania N, Yazan LS, Ismail IS, et al (2013). Dillenia Suffruticosa extract inhibits proliferation of human breast cancer cell lines (MCF-7 and MDA-MB-231) via induction of G2/M arrest and apoptosis. Molecules, 18, 13320-39.

Cho KS, Elizondo LI, Boerkoel CF (2004). Advances in chromatin remodeling and human disease. Curr Opin Genet Dev, 14, 308-15.

Choi EJ, Kim GH (2013). O-desmethylangolensin inhibits the proliferation of human breast cancer MCF-7 cells by inducing apoptosis and promoting cell cycle arrest. Oncol Lett, 6, 1784-8.

Cui Y, Niu A, Pestell R, et al (2006). Metastasis-associated protein 2 is a repressor of estrogen receptor alpha whose overexpression leads to estrogen-independent growth of human breast cancer cells. Mol Endocrinol, 20, 2020-35.

Ding H, Wu YL, Wang YX, Zhu FF, et al (2014). Characterization of the microRNA expression profile of cervical squamous cell carcinoma metastases. Asian Pac J Cancer Prev, 15, 1675-9.

Erdogan B, Cicin I (2014). Medical treatment of breast cancer bone metastasis: from bisphosphonates to targeted drugs. Asian Pac J Cancer Prev, 15, 1503-10.

Fagan-Solis KD, Schneider SS, Pentecost BT, et al (2013). The RhoA pathway mediates MMP-2 and MMP-9-independent invasive behavior in a triple-negative breast cancer cell line. $J$ Cell Biochem, 114, 1385-94.

Fearon ER (2003). Connecting estrogen receptor function, transcriptional repression, and E-cadherin expression in breast cancer. Cancer Cell, 3, 307-10.

Fidler IJ (1990). Critical factors in the biology of human cancer metastasis: twenty-eighth G.H.A. Clowes memorial award lecture. Cancer Res, 50, 6130-8.

Fujita N, Jaye DL, Kajita M, Geigerman C, Moreno CS, Wade PA (2003). MTA3, a Mi-2/NuRD complex subunit, regulates an invasive growth pathway in breast cancer. Cell, 113, 207-19.

Kumar R (2003). Another tie that binds the MTA family to breast cancer. Cell, 113, 142-3.

Kumar R, Wang RA, Bagheri-Yarmand R (2003). Emerging roles of MTA family members in human cancers. Semin Oncol, 30, 30-7.

Luo J, Su F, Chen D, Shiloh A, Gu W (2000). Deacetylation of p53 modulates its effect on cell growth and apoptosis. Nature, 408, 377-81.

Matsusue K, Takiguchi S, Toh Y, KonoA (2001). Characterization of mouse metastasis-associated gene 2: genomic structure, nuclear localization signal, and alternative potentials as transcriptional activator and repressor. DNA Cell Biol, 20, 603-11.

Puzovic V, Brcic I, Ranogajec I, Jakic-Razumovic J (2014). Prognostic values of ETS-1, MMP-2 and MMP-9 expression and co-expression in breast cancer patients. Neoplasma, 61, 439-47.

Radenkovic S, Konjevic G, Jurisic V, Karadzic K, Nikitovic M, Gopcevic K (2014). Values of MMP-2 and MMP-9 in tumor tissue of basal-like breast cancer patients. Cell Biochem Biophys, 68, 143-52.

Roberts CW, Orkin SH (2004). The SWI/SNF complexchromatin and cancer. Nat Rev Cancer, 4, 133-42.

Shaukat U, Ismail M, Mehmood N (2013). Epidemiology, major risk factors and genetic predisposition for breast cancer in the Pakistani population. Asian Pac J Cancer Prev, 14, 5625-9.

Toh Y, Kuwano H, Mori M, Nicolson GL, Sugimachi K (1999). Overexpression of metastasis-associated MTA1 mRNA in invasive oesophageal carcinomas. Br J Cancer, 79, 1723-6.

Toh Y, Oki E, Oda S et al (1997). Overexpression of the MTA1 gene in gastrointestinal carcinomas: correlation with invasion and metastasis. Int J Cancer, 74, 459-63.

Toh Y, Pencil SD, Nicolson GL (1994). A novel candidate metastasis-associated gene, mta1, differentially expressed in highly metastatic mammary adenocarcinoma cell lines. cDNA cloning, expression and protein analyses. $J$ Biol Chem, 269, 22958-63.

Tong JK, Hassig CA, Schnitzler GR, Kingston RE, Schreiber SL (1998). Chromatin deacetylation by an ATP-dependent nucleosome remodelling complex. Nature, 395, 917-21.

Wu JQ, Li YY, Ren JC, Zhao R, Zhou Y, Gao ES (2014). Induced abortion and breast cancer: results from a population-based case control study in china. Asian Pac J Cancer Prev, 15, 3635-40.

Zhang H, Stephens LC, Kumar R (2006). Metastasis tumor antigen family proteins during breast cancer progression and metastasis in a reliable mouse model for human breast cancer. Clin Cancer Res, 12, 1479-86.

Zhou C, Ji J, Cai Q, et al (2013). MTA2 promotes gastric cancer cells invasion and is transcriptionally regulated by $\mathrm{Sp} 1 . \mathrm{Mol}$ Cancer, 12, 102. 